\title{
Purification of thrombospondin receptor (CD36) from human platelet membrane
}

\author{
Joongho Kim, ${ }^{1}$ Kyoungho Suk, ${ }^{1}$ Na-Young \\ Park $^{1}$ and Seung-Ho Kim ${ }^{1,2}$

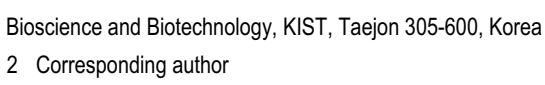

\begin{abstract}
Thrombospondin receptor (CD36) has been recently identified in platelets and various cell lines as the receptor for thrombospondin, an adhesive protein required for irreversible aggregation of platelets as well as other adhesive processes. Thrombospondin receptor, one of major glycosylated platelet membrane proteins, is thought to play an important role as a cell adhesion molecule in blood coagulation system as well as intercellular signaling. In this work, thrombospondin receptor was purified to homogeneity from human platelet by wheat germ agglutinin (WGA)-affinity chromatography and size exclusion chromatography on Ultrogel-AcA44. The molecular weight of the purified thrombospondin receptor was about $88 \mathrm{kDa}$ on SDS-PAGE and its identity was confirmed by immunoblot analysis and immunodiffusion assay.
\end{abstract}

Keywords: CD36, glycoprotein IV (GPIV), platelets, thrombosis, thrombospondin receptor

\section{Introduction}

Thrombospondin receptor, known as GPIIlb, glycoprotein IV (GPIV) or CD36, is a highly glycosylated platelet membrane protein that is not related to a known gene family (Huang et al., 1991). Thrombospondin receptor or CD36 has been found in monocytes, endothelial cells, melanoma cells, and nucleated erythroid cells (Knapp et al., 1989). The cDNA of CD36 has been cloned and sequenced from placenta, and deduced amino acid sequences predict an extracellular domain of 438 amino acid residues, a transmembrane domain of 24 residues, and a short cytoplasmic tail of 6 residues
(Knapp et al., 1989).

The CD36 has been recently identified in platelets and various cell lines as the receptor for thrombospondin. Thrombospondin is an adhesive protein that is required for irreversible aggregation of platelets as well as other adhesive processes (Asch et al., 1987). Thrombospondin is secreted by platelets, endothelial cells as well as macrophages, and plays an important role in the consolidation of hemostatic plugs by being incorporated into fibrin clots. It has been shown that CD36 binds to collagen type I fibrils, and antibody against CD36 completely blocks collagen-induced platelet aggregation (Diaz-Ricart et al., 1993; Saelman et al., 1994). The CD36 has been also implicated in the adherence of Plasmodium falciparum-infected erythrocytes to the endothelium (Howard and Gilladoga, 1989). As suggested by these previous studies CD36 may have important functions as a cell adhesion molecule in blood coagulation system as well as intercellular signaling. In the current work, we purified human CD36 from platelet membrane by wheat germ agglutinin (WGA) affinity chromatography combined with size exclusion chromatography on Ultrogel-AcA44. Although partial biochemical characterization of CD36 has been documented, purified human CD36 would allow one to further characterize the protein.

\section{Materials and Methods}

\section{Materials}

Triton X-114, Triton X-100, thrombin, trypsin, soybean trypsin inhibitor, 4-chloro-1-naphthol, and anti-mouse antibody conjugated with horseradish peroxidase were purchased from Sigma (St. Louis, MO). WGA-sepharose was obtained from Vector Laboratories (Burlingame, CA), Ultrogel AcA-44 from LKB (Gaithersburg, MD), and CD36 mouse monoclo-nal antibody from Pharmingen (San Diego, CA).

\section{Preparation of human platelet}

Human platelets were isolated from platelet concentrates by differential centrifugation on Ficoll (Pharmacia) according to the method of Anderson and Gahmberg, 1978. Microscopic observation showed that the proportion of the platelets in this cell preparation was more than $99.5 \%$.

\section{Purification of CD36}

Intact platelets were first proteolyzed to remove proteolysis-sensitive membrane proteins by incubating 
with trypsin at $20^{\circ} \mathrm{C}$ for $20 \mathrm{~h}$ followed by neutralization with soybean trypsin inhibitor. Washed platelets were then lysed by sonication and membrane fractions $(62.4$ $\mathrm{mg}$ of total protein) were solubilized in $30 \mathrm{ml}$ of $1 \%$ Triton X-114 containing $50 \mathrm{mM}$ Tris- $\mathrm{HCl}(\mathrm{pH} 7.4), 5 \mathrm{mM}$ EGTA, and $0.5 \mathrm{mM}$ phenylmethylsulfonyl fluoride as described (Tandon et al., 1989) with slight modifications. Insoluble materials were removed by centrifugation at $100,000 \mathrm{~g}$ for $30 \mathrm{~min}$. The Triton solution was warmed at $37^{\circ} \mathrm{C}$ for $15 \mathrm{~min}$ for phase separation and then centrifuged at $1,800 \mathrm{~g}$ for $10 \mathrm{~min}$. Detergent phase was loaded onto WGA-Sepharose column equilibriated with the buffer containing $50 \mathrm{mM}$ Tris- $\mathrm{HCl}, 5 \mathrm{mM}$ EGTA (pH 7.4) and $0.1 \%$ Triton X-100. Absorbed CD36 was then eluted with 3 column volumes of $250 \mathrm{mM} \mathrm{N}$-acetylglucosamine. Fractions containing proteins were combined, dialyzed, and concentrated using an Amicon filtration assembly. For further purification concentrates were made $1 \%$ with SDS, warmed at $80^{\circ} \mathrm{C}$ for $2 \mathrm{~min}$, cooled, and loaded onto an Ultrogel AcA-44 column equilibriated in $50 \mathrm{mM}$ Tris- $\mathrm{HCl}(\mathrm{pH}$ 7.4), 1 mM EGTA, $0.05 \%$ Triton $X-100$, and $0.05 \%$ SDS, which was subsequently eluted with the same buffer at a flow rate of $12 \mathrm{ml} / \mathrm{h}$, and fractions of $3 \mathrm{ml}$ collected. Fractions showing absorbance at $280 \mathrm{~nm}$ were analyzed by SDS-PAGE and those containing CD36 were pooled, dialyzed against $50 \mathrm{mM}$ Tris- $\mathrm{HCl}$ (pH 7.4), $150 \mathrm{mM} \mathrm{NaCl}$, and concentrated by Amicon filtration.

\section{Electrophoresis and immunoblot analysis}

Polyacrylamide gel electrophoresis in the presence of SDS was carried out by Laemmli system (Laemmli, 1970 ) on $7 \%$ polyacrylamide slab gel. After electrophoresis, the gels were stained with either Coomassie blue or periodic acid-Schiff reagent. For immunoblot analysis, proteins separated by electrophoresis were transferred to the membrane. And, the membrane was sequentially incubated with blocking solution of $2 \%$ BSA, anti-CD36 monoclonal antibody, anti-mouse antibody conjugated with horseradish peroxidase, and chromogenic substrate, 4-chloro-1-naphthol.

\section{Immunodiffusion assay}

$1.5 \%$ agar gels were poured onto slides and allowed to set. Wells are then punched in the gel and $10 \mu \mathrm{l}$ of test solutions of antigens and antibodies were added to the wells. After solutions diffuse out, formation of precipitation lines was examined.

\section{Results and Discussion}

As outlined in the purification scheme (Figure 1), human platelet membrane was first extensively trypsinized to remove proteolysis-sensitive surface glycoproteins. This is based on the previous observation that CD36 is resistant to proteolysis in intact platelets (Okumura and Jamieson, 1976). Trypsinized platelet membrane was then solubilized using Triton $\mathrm{X}-114$ and detergent phase was subjected to WGA-affinity chromatography (Figure 2). Lectins have been shown to specifically recognize and bind to certain glycoproteins and this specific interaction between lectins and glycoproteins has been successfully used in the purification of platelet membrane glycoproteins (Clemetson et al., 1981, Leung et al., 1981). A combination of various immobilized lectins with different binding characteristics was revealed to be very effective for the purification of glycoproteins.

Fractions eluted from WGA-affinity chromatography were analyzed by SDS-PAGE (Figure 3). Fractions 38 and 39 appeared to contain CD36 as a major protein and some lower molecular weight components. The major protein was first assumed as CD36 and further purifications proceeded, because the protein has the molecular weight similar to a known CD36 and two proteins were purified by similar procedures. The identity of the protein was confirmed after the final purification step. The minor contaminants in the fractions of WGAaffinity chromatography were removed by size exclusion chromatography on Ultrogel-AcA44. Again, CD36 was eluted and fractions electrophoresed (Figure 4). Fractions showing a single band of purified CD36 on a gel were pooled and concentrated for further analysis.

Purified CD36 was subjected to immunoblot analysis to confirm the identity and to test the antigenicity of the protein (Figure 5). The purified protein of $88 \mathrm{kDa}$ was specifically recognized by anti-CD36 antibody. Proteins with higher molecular weights appear to be those that cross-reacted with anti-CD36 antibody. We currently cannot explain the nature of these proteins. Integrity of antigenic properties of purified CD36 was further

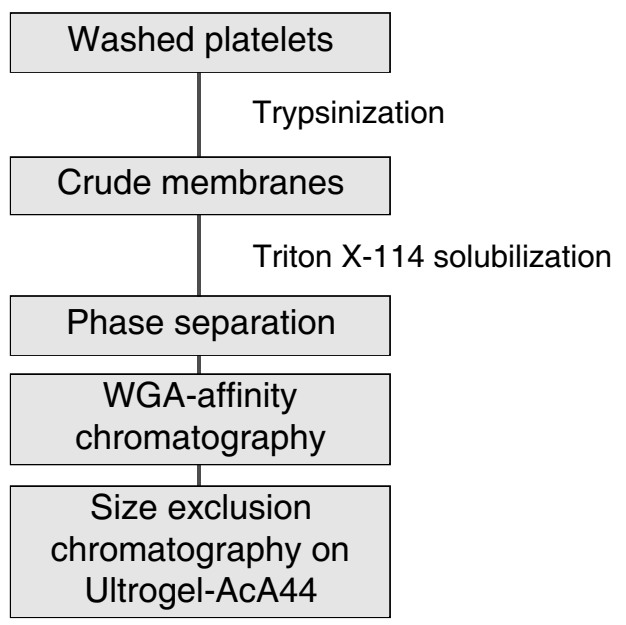

Figure 1. Purification scheme of CD36. 


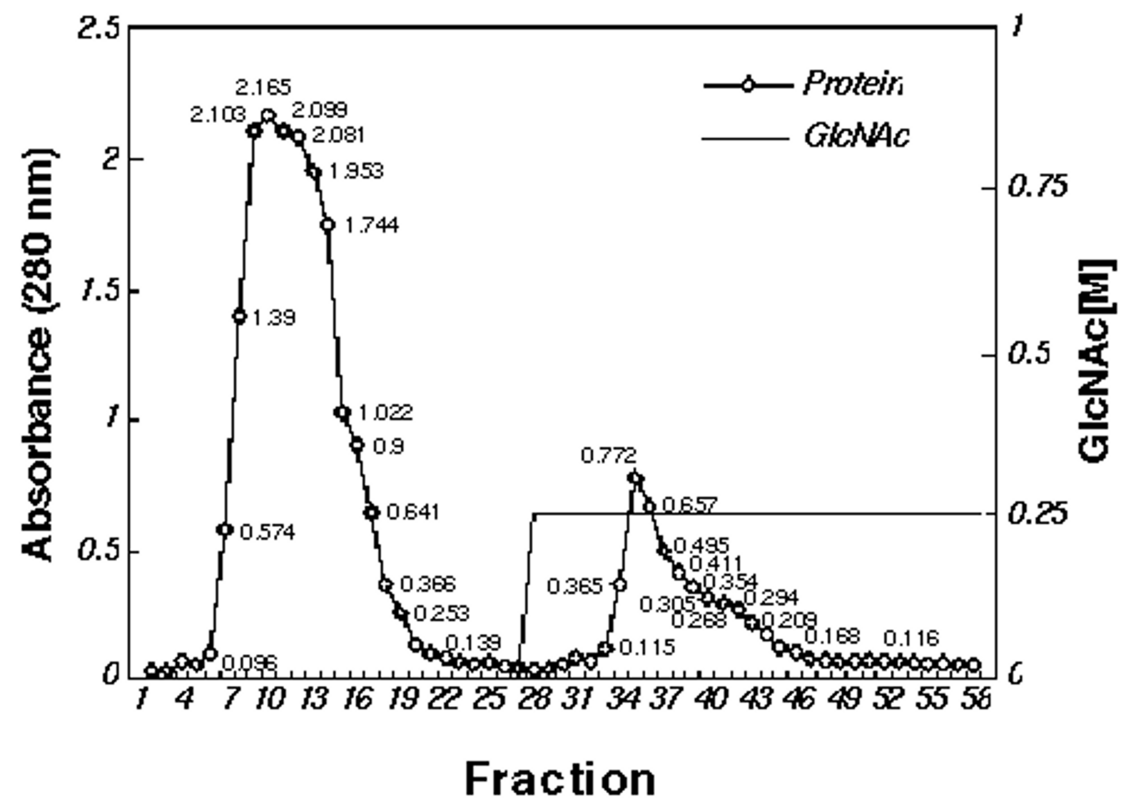

Figure 2. WGA-affinity chromato-graphy. Solubilized membranes of trypsinized platelets were loaded onto WGA-affinity column $(1.2 \times 12$ $\mathrm{cm})$. The column was first eluted with the buffer containing $50 \mathrm{mM}$ Tris $\mathrm{HCl}, 5$ mM EGTA (pH 7.4) and $0.1 \%$ Triton $\mathrm{X}-100$, then absorbed proteins were eluted with the same buffer containing 250 $\mathrm{mM} \mathrm{N}$-acetylglucosamine (GlcNAc). Fractions were collected and absorbance measured at $280 \mathrm{~nm}$. Solid line indicates absorbance values of each fraction and dotted line represents the time point where GICNAc was added for the elution. The first peak of the solid line appears to be unbound proteins and the second peak seems to contain CD36.

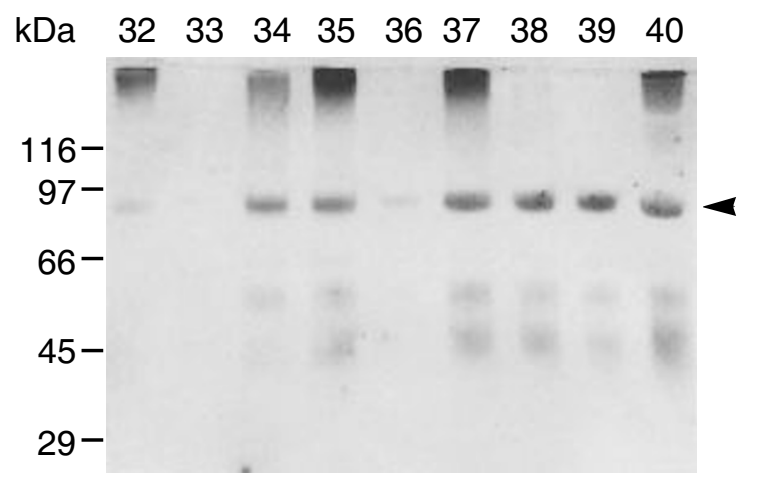

Figure 3. SDS-PAGE of fractions eluted from WGA-Sepharose column. Fraction eluted from WGA-Sepharose column was electrophoresed on 7\% SDS-PAGE and stained with Coomassie blue. Lane numbers correspond to fraction numbers in Figure 2. The arrowhead indicates the protein that was assumed as CD36. The intensity of $88-\mathrm{kDa}$ band in the fraction 36 is relatively weak because most of samples of this fraction was lost by mistake during experiment. This, however, did not significantly affect general purification scheme.

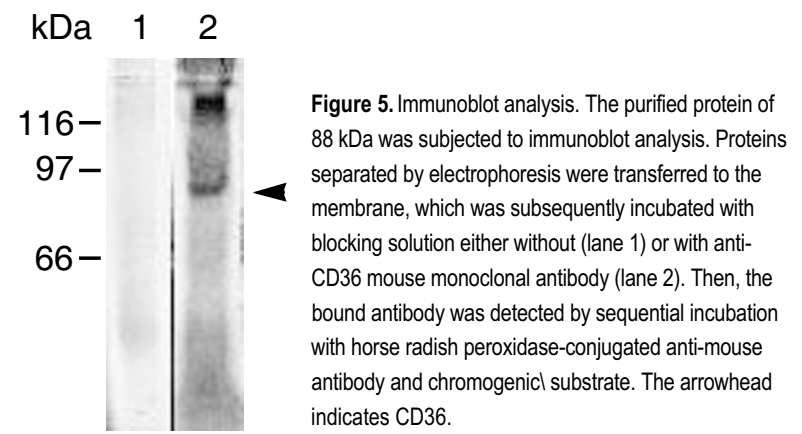

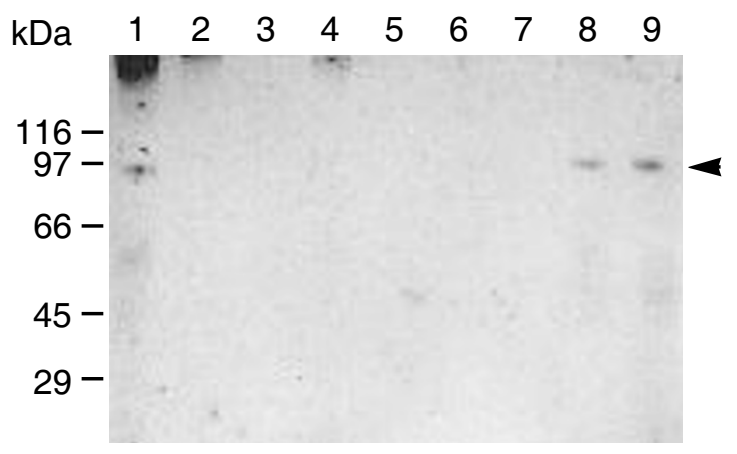

Figure 4. SDS-PAGE of fractions eluted from Ultrogel AcA-44 column. Pooled fractions containing partially purified CD36 (fractions 34 to 40 in Figure 3 ) were passed over Ultrogel AcA-44 column (fractionation range of $10-130 \mathrm{kDa}$ ), and fractions again analyzed by SDS-PAGE. Representative 9 fractions (lane 1 to 9 ) were electrophoresed on $7 \%$ gel and stained with Coomassie blue. The arrowhead indicates the protein that was assumed as $\mathrm{CD} 36$.
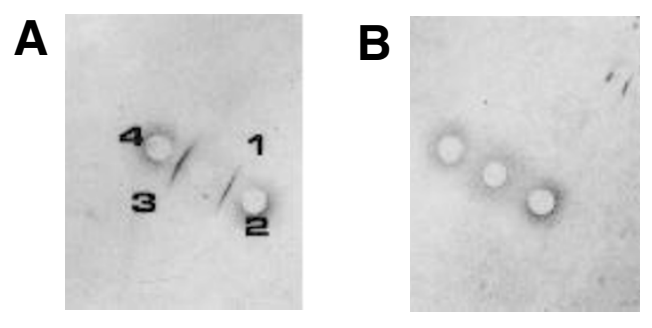

Figure 6. Immunodiffusion assay with purified CD36 and thrombin. (A). Center well contained $5 \mu \mathrm{g}$ of CD36 antibody and outside wells contained $7.5 \mu \mathrm{g}$ of either purified CD36 (well 2 and 4), or thrombin (well 1 and 3). Only purified CD36 (well 2, 4) formed precipitation lines by complexing with anti-CD36 antibody. (B). Center well contained 5 $\mu_{\mathrm{g}}$ of thrombin antibody and outside wells contained $7.5 \mu_{\mathrm{g}}$ of purified CD36. 
demonstrated by immunodiffusion assay (Figure 6). The purified CD36 protein showed a distinct precipitation band by forming complex with CD36 antibody. Thrombin, another important blood protein for platelet functions, was used as a negative control and did not react with CD36 antibody.

Taken together, CD36 was purified to apparent homogeneity by WGA-affinity chromatography and size exclusion chromatography. Although CD36 has previously been purified using lectin columns (Tandon et al., 1989, Tsuji and Osawa, 1986), the procedure employed in the current study is much simpler and time-saving without compromizing the purity and yield of the protein. The protein isolated by Tsuji and Osawa was described as CD36, but the protein showed proteolytic resistance, amino acid and carbohydrate analyses different from a known CD36 later purified (Tandon et al., 1989). Compared to purification procedures employed by Tandon group (Tandon et al., 1989), our current method does not involve DEAE chromatography or lectinprecipitation step between phase separation of Triton X114 solubilized membranes and WGA-affinity chromatography (see purification scheme in Figure 1). The purification was initiated from $62.4 \mathrm{mg}$ of total protein in platelets and the final recovery yield was 0.12 $\%$, which is comparable to previously described methods (Tandon et al., 1989; Tsuji and Osawa, 1986).

Some of important functions of CD36 as a cell adhesion molecule have been previously reported (Asch et al., 1987; Howard and Gilladoga; 1989, Diaz-Ricart et al., 1993; Saelman et al., 1994). Recent findings on the involvement of CD36 in thrombotic thrombocytopenic purpura (TTP) and other disorders of hematopoietic system indicate its pathological implications and yet other critical functions (Byrnes and Moake, 1986; Lian et al., 1991; Siddiqui and Lian, 1992). Now, purified CD36 could be utilized for further characterization of the protein in biochemical as well as clinical aspects. For instance, we evaluated the possibility of CD36 binding to $37-\mathrm{kDa}$ platelet agglutinating protein (PAP p37) found in TTP patient plasma. Although there has been a claim that platelet aggregation in TTP may be mediated by direct interaction between CD36 and PAP p37 (Lian et al., 1991), we were not able to confirm the results (data not shown). If indeed, however, platelet agglutination in TTP patient occurs through interaction between CD36 and PAP p37, inhibitors that block the interaction of two proteins can be designed for therapeutic purpose. For such applications, purified CD36 can be used for the generation of anti-CD36 antibody or searching for peptides that has such inhibitory effects. Elucidation of pathophysiological role of CD36 in TTP and other disorders is certainly an intriguing research area that awaits further works.

\section{Acknowledgement}

This work was supported by a grant from Korean Ministry of Science and Technology (HAN project, HS1140).

\section{References}

Andersson, L. C. and Gahmberg, C. G. (1978) Surface glycoproteins of human white blood cells. Analysis by surface labeling. Blood 52: 57-67

Asch, A. S., Barnwell, J., Siverstein, R. L. and Nachman, R. L. (1987) Isolation of the thrombospondin membrane receptor. J. Clin. Invest. 79: 1054-1061

Byrnes, J. J. and Moake, J. L. (1986) Thrombotic thrombocytopenic purpura and the haemolytic-uraemic syndrome: evolving concepts of pathogenesis and therapy. Clinics Haematol. 15: 413-442

Clemetson, K. J., Naim, H. Y. and Luscher, E. F. (1981) Relationship between glycocalicin and glycoprotein lb on human platelets. Proc. Natl. Acad. Sci. USA 78: 2712-2716

Diaz-Ricart, M., Tandon, N. N., Carretero, M., Ordinas, A., Bastida, E., and Jamieson, G. A. (1993) Platelet lacking functional CD36 (glycoprotein IV) show reduced adhesion to collagen in flowing whole blood. Blood 82: 491-496

Howard, R. J. and Gilladoga, A. D. (1989) Molecular studies related to the pathogenesis of cerebral malaria. Blood 74: 2603-2618

Huang, M.-M., Bolen, J. B., Branwell, J. W., Smith, S. J., and Brugge, J. S. (1991) Membrane glycoprotein IV (CD36) is physically associated with the Fyn, Lyn, \& Yes protein-tyrosine kinases in human platelets. Proc. Natl. Acad. Sci. U.S.A. 88: 7844-7848

Knapp, W., Dorken, B., and Rieber, P. (1989) CD antigens 1989. Blood 74: 1448-1450

Laemmli, U. K. (1970) Cleavage of structural proteins during the assembly of the head of bacteriophage T4. Nature 227: 680-685

Leung, L. K., Kinoshita, T., and Nachman, R. L. (1981) Isolation, purification, and partial characterization of platelet membrane glycoproteins Ilb and IIla. J. Biol. Chem. 256: 1994-1997

Lian, E. C.-Y., Siddiqui, F. A., Jamieson, G. A., and Tandon, N. N. (1991) Platelet agglutinating protein $\mathrm{p} 37$ causes platelet agglutination through its binding to membrane glycoprotein IV. Thromb. Haemosta.65: 102-106

Okumura, T. and Jamieson, G. A. (1976) Platelet glycocalicin I. Orientation of glycoproteins of the human platelet surface. J. Biol. Chem. 251: 5944-5949

Saelman, E. U. M., Kehrel, B., Hese, K. M., deGroot, P. G., Sixma, J. J., and Nieuwenhuis, H. K. (1994) Platelet adhesion to collagen and endothelial cell matrix under flow conditions is not dependent on platelet glycoprotein IV. Blood 83: 3240-3244

Siddiqui, F. A. and Lian, E. C.-Y. (1992) Platelet-agglutinating protein p37 from a thrombotic thrombocytopenic purpura plasma forms complex with platelet membrane glycoprotein IV (CD36). Biochem. Intern. 27: 485-496

Tandon, N. N., Lipsky, R. H., Burgess, W. H., and Jamieson, G. A. (1989) Isolation and characterization of platelet glycoprotein IV (CD36). J. Biol. Chem. 264: 7570-7575

Tsuji, T. and Osawa, T. (1986) Purification and chemical characterization of human platelet membrane glycoprotein IV. J. Biochem. 100: 1077-1085 